\title{
A COMPLETE Look at the Use of IRAS Emission Maps to Estimate Extinction and Dust Temperature
}

\section{Citation}

Schnee, Scott L., Naomi A. Ridge, Alyssa A. Goodman, and Jason G. Li. 2005. "A COMPLETE Look at the Use ofIRASEmission Maps to Estimate Extinction and Dust Temperature." The Astrophysical Journal 634 (1): 442-50. https://doi.org/10.1086/491729.

\section{Permanent link}

http://nrs.harvard.edu/urn-3:HUL.InstRepos:41393404

\section{Terms of Use}

This article was downloaded from Harvard University's DASH repository, and is made available under the terms and conditions applicable to Other Posted Material, as set forth at http:// nrs.harvard.edu/urn-3:HUL.InstRepos:dash.current.terms-of-use\#LAA

\section{Share Your Story}

The Harvard community has made this article openly available.

Please share how this access benefits you. Submit a story.

Accessibility 


\title{
A COMPLETE LOOK AT THE USE OF IRAS EMISSION MAPS TO ESTIMATE EXTINCTION AND DUST TEMPERATURE
}

\author{
Scott L. Schnee, Naomi A. Ridge, Alyssa A. Goodman, and Jason G. Li \\ Harvard-Smithsonian Center for Astrophysics, 60 Garden Street, Cambridge, MA 02138 \\ Received 2005 April 28; accepted 2005 July 30
}

\begin{abstract}
We have created new dust temperature and column density maps of Perseus, Ophiuchus, and Serpens using 60 and $100 \mu \mathrm{m}$ data from the Improved Reprocessing of the IRAS Survey (IRIS) recalibration of Infrared Astronomical Satellite $(I R A S)$ data. We describe an optimized method for finding the dust temperature, emissivity spectral index, and optical depth using optical and near-infrared extinction maps. The creation of these temperature and extinction maps (covering tens of square degrees of molecular clouds) is one of the first results from the ongoing Coordinated Molecular Probe Line Extinction Thermal Emission (COMPLETE) Survey of Star-Forming Regions. However, while the extinctions derived from the IRIS emission maps are globally accurate, we warn that far-infrared emission is not a good proxy for extinction on the scale of 1 pixel $\left(\sim 5^{\prime}\right)$. In addition to describing the global dust properties of these clouds, we have found two particularly interesting features in the column density and temperature maps. In the Ophiuchus dark cloud complex, the new dust temperature map shows a little-known warm (25 K) dust ring with a 2 pc diameter. This shell is approximately centered on the B star $\rho$ Ophiuchus, $1^{\circ}$ north of the well-studied $\rho$ Oph star-forming cluster. In Perseus, the column density map shows a 10 pc diameter ring, a feature not apparent in the filamentary chain of clouds seen in molecular gas. These rings are further discussed in detail in our companion papers.

Subject headings: dust, extinction — ISM: clouds — surveys
\end{abstract}

Online material: color figures

\section{INTRODUCTION}

The goal of the COMPLETE Survey is to use a carefully chosen set of observing techniques to fully sample the density, temperature, and velocity structure of three of the five large starforming complexes observed in the NASA-sponsored Spitzer Legacy Survey "From Molecular Cores to Planet-forming Disks" (cores to disks [c2d]) described in Evans et al. (2003).

The c2d Survey, started in late 2003, is producing highresolution infrared spectroscopy and near- through far-infrared (NIR, FIR) images of each of its 5 pc scale target complexes. COMPLETE is providing fully sampled millimeter spectral line, extinction, and thermal emission maps for the same regions, at arcminute resolution or better (Goodman 2004). Phase II of COMPLETE, begun recently, provides higher resolution observations using the same suite of techniques for a large subset of the high-density cores evident at lower resolution.

In this paper we present new temperature and extinction maps of the Perseus, Serpens, and Ophiuchus star-forming regions, produced from 60 and $100 \mu \mathrm{m}$ flux density maps obtained from IRIS and normalized using optical/NIR extinction maps generated by J. Alves et al. (2005, in preparation) and Cambrésy (1999). Previous all-sky thermal dust emission maps by Schlegel et al. (1998, hereafter SFD98) include these regions. However, the SFD98 maps were based on low-resolution temperature data and optimized for low-extinction regions. It has been established that the SFD98 maps lose accuracy at $A_{V}>0.5 \mathrm{mag}$ (Arce \& Goodman 1999a; Cambrésy et al. 2005), so they are not adequate for mapping column density in the high-extinction areas targeted in the COMPLETE survey. We show here that reanalysis of the IRAS data can yield column density and color temperature maps that are more accurate at high and low levels of extinction on the large scale, although their use on the scale of the data $\left(\sim 5^{\prime}\right)$ is more limited.

In $\oint 2$ we describe the IRIS flux measurements and NIR/ optical-based extinction maps. In $\S \S 3$ and 4 we present and ex- plain the equations used to determine the dust optical depth and conversion to visual extinction and the parameters needed in those equations. In $\S 5$ we compare the results of our analysis technique to those from other extinction tracers. The results are summarized in $\S 6$.

\section{DATA}

\subsection{IRAS/IRIS}

IRIS $^{1}$ images of flux density at 60 and $100 \mu \mathrm{m}$ were obtained for each of our three target regions from the IRIS Web site (Miville-Deschênes \& Lagache 2005). Image details are given in Table 1. The maps are in units of megajansky per steradian, are made with gnomic projection, and have spatial resolution smoothed to 4.3 at $100 \mu \mathrm{m}$.

IRIS data offer excellent correction for the effects of zodiacal dust and striping in the images and also provide improved gain and offset calibration. Earlier releases of the IRAS data did not have the appropriate zero-point calibration, which can have serious consequences on the derived dust temperature and column density (Arce \& Goodman 1999a, 1999b). To test the zero-point calibration of the IRIS data, we allowed two free parameters in the fit (one each for the 60 and $100 \mu \mathrm{m}$ zero points) to convert IRIS fluxes to visual extinction (see $\S 4$ ). We find that values of the free parameters are consistent with zero and thus provide independent evidence that the zero-point calibrations of the IRIS data at 60 and $100 \mu \mathrm{m}$ are correct.

\subsection{Optical and NIR Extinction Maps}

Here we describe the NIR and optical extinction maps used to recalibrate the IRIS maps presented in this paper. We have obtained extinction maps of Serpens and Ophiuchus from Cambrésy (1999), which are based on an optical star counting method with

\footnotetext{
${ }^{1}$ IRIS comprises a machine-readable atlas of the sky in the four IRAS bands at $12,25,60$, and $100 \mu \mathrm{m}$
} 
TABLE 1

IRIS Image Details

\begin{tabular}{|c|c|c|c|}
\hline Region & $\begin{array}{l}\text { Center R.A. } \\
\text { (deg) }\end{array}$ & $\begin{array}{c}\text { Center Decl. } \\
\text { (deg) }\end{array}$ & $\begin{array}{c}\text { Image Size } \\
\text { (deg) }\end{array}$ \\
\hline Perseus .......................... & 54.30 & 31.85 & $6.6 \times 4.5$ \\
\hline Perseus ring ..................... & 54.91 & 31.87 & $1.8 \times 1.8$ \\
\hline В5 ................................. & 56.90 & 32.85 & $0.8 \times 0.8$ \\
\hline IC 348 & 55.94 & 31.99 & $1.0 \times 1.0$ \\
\hline NGC $1333 \ldots \ldots \ldots \ldots \ldots \ldots$ & 52.16 & 31.35 & $1.0 \times 1.0$ \\
\hline Ophiuchus ......................... & 247.95 & -24.00 & $6.9 \times 6.8$ \\
\hline Serpens .................... & 277.00 & 0.50 & $3.0 \times 3.0$ \\
\hline
\end{tabular}

Note.-All positions are given in equatorial J2000.0 equinox coordinates.

variable resolution (but we regrid these maps to the constant resolution of the IRIS images). The optical photometry data used by Cambrésy come from the US Naval Observatory Precision Measuring Machine (USNO-PMM) catalog (Monet 1996). Extinction maps of Perseus and Ophiuchus have been constructed from the Two Micron All Sky Survey (2MASS) point-source catalog as part of COMPLETE by J. Alves et al. (2005, in preparation) using the "NICER" algorithm, which is a revised version of the NICE method described in Lada et al. (1994) and Lombardi \& Alves (2001). NICE and NICER combine direct measurements of NIR color excess and certain techniques of star counting to derive mean extinctions and map the dust column density distribution through a cloud (Lombardi \& Alves 2001). The 2MASS survey provides the NIR $J, H$, and $K_{s}$ colors of background stars that have been reddened by the molecular cloud. With these measured colors, and knowledge of the intrinsic colors of these stars (measured in a nearby, nonreddened control field), the amount of obscuring material along the line of sight to each star can be determined. In NICER, the extinction values are calculated for a fixed resolution, which means that uncertainties vary from pixel to pixel. Maps of uncertainty for the regions considered are shown in J. Alves et al. (2005, in preparation).

In the Ophiuchus molecular cloud, we have both 2MASS/ NICER and optical star counting-based extinction maps. It is important to note that the two methods do not give equivalent extinctions. A plot of the optical versus NIR extinctions is shown in Figure 1. A linear fit to the data shows that the slope of the line relating the two quantities is very close to unity (as expected), but there is an offset of roughly $0.71 \mathrm{mag}$ of visual extinction, with the 2MASS-derived data points being systematically higher than the optically derived extinctions. The $1 \sigma$ scatter on the leastsquares fit between the two methods is $0.7 \mathrm{mag}$ in $A_{V}$. Because these methods produce such different results, care must be taken when using any one extinction map as a model to determine dust properties as described in $\S 3$. In Ophiuchus, where we have both optical and NIR extinction maps, we run the global fitting algorithm for each data set separately and report both sets of results in Table 2.

In Perseus we only have a NIR-based extinction map, and in Serpens we only have an optically derived extinction map, so no intercomparison of the calibration methods is possible for these clouds. We expect that the 2MASS-derived extinctions are more accurate than the star counting method of Cambrésy, although in both cases the zero-point calibration can be difficult to assess. The optical star counting method chooses the average minimum value in the map as the "zero level" of extinction (Cambrésy 1999), and the NICER method uses the average minimum $\mathrm{H}-$ $K$ color to determine the minimum extinction in the map. Both methods rely on the minimum in their map actually cor-

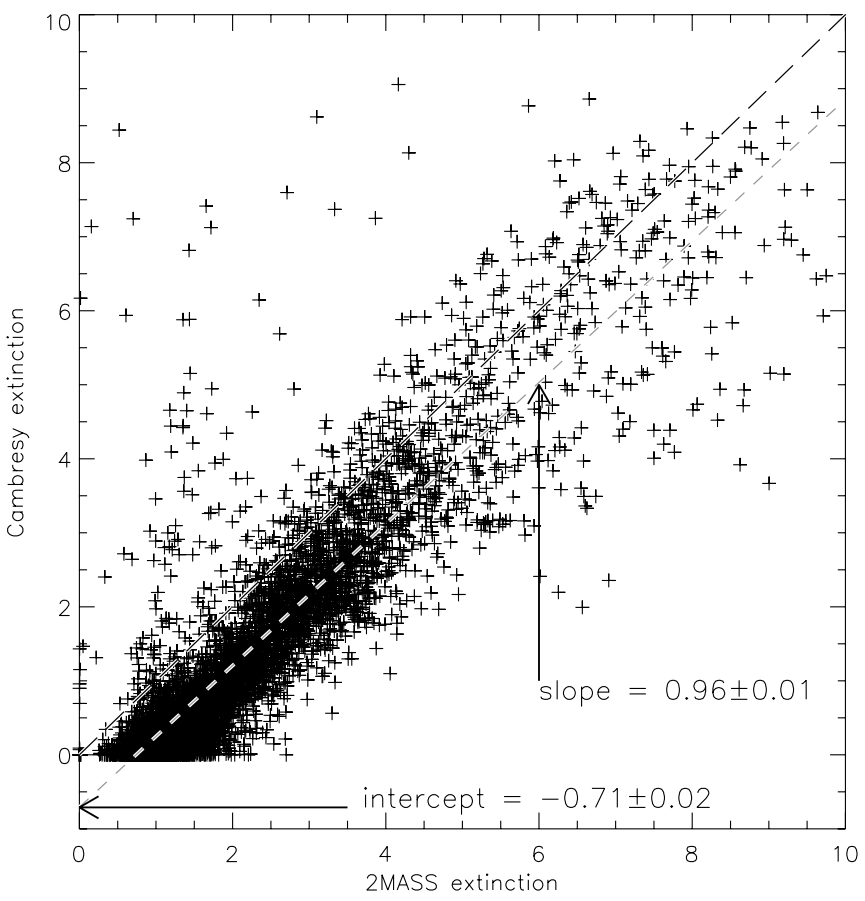

FIG. 1.-Scatter plot of the Ophiuchus optical star counting (Cambrésy 1999) and NIR color excess method (J. Alves et al. 2005, in preparation) extinctions in Ophiuchus. The short-dashed line is the best-fit line to the data sets, with the equation shown at the bottom of the image. The long-dashed (1:1) line is there only to guide the eye. Nearly $60 \%$ of the data points have 2 MASS extinctions less than 2. [See the electronic edition of the Journal for a color version of this figure.]

responding to zero extinction, which if untrue will cause both methods to underestimate the true extinction. In Ophiuchus, because the NICER algorithm gives higher extinctions, it is likely that the optical star counting algorithm has underestimated the true extinction by approximately $0.71 \mathrm{mag}$. Our decision to trust the NIR-based extinctions over the star counting extinctions is supported by discussion with Professors L. Cambrésy \& J. Alves (2005, private communication). To account for the $0.71 \mathrm{mag}$ offset, we have added $0.71 \mathrm{mag}$ to the extinction values derived by Cambrésy (1999) in our optically calibrated calculations for Ophiuchus in $\S \S 3$ and 4.

We expect the Serpens extinction map to suffer from the same zero level problem as the Ophiuchus optical extinction map and therefore include a free parameter in the fit between the optical extinction map and the IRIS emission maps to account for this

TABLE 2

Best-Fit IRAS/Dust Parameters

\begin{tabular}{|c|c|c|c|c|}
\hline Cloud & $\delta$ & $\omega$ & $X$ & $\begin{array}{l}A_{V} \text { Offset } \\
\left(\operatorname{mag} A_{V}\right)\end{array}$ \\
\hline Perseus …….................... & 0.32 & 0.0180 & 1100 & $\ldots$ \\
\hline B5 ................................ & 0.38 & 0.0140 & 1200 & $\ldots$ \\
\hline IC 348 & 0.33 & 0.0160 & 1000 & $\ldots$ \\
\hline 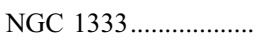 & 0.64 & 0.0010 & 1300 & $\ldots$ \\
\hline c2d area & 0.36 & 0.0140 & 1200 & $\ldots$ \\
\hline Ring ............................ & 0.38 & 0.0150 & 990 & $\ldots$ \\
\hline Ophiuchus $^{\mathrm{a}} \ldots \ldots \ldots \ldots \ldots$ & 0.46 & 0.0016 & 510 & $\ldots$ \\
\hline Ophiuchus $^{\mathrm{b}} \ldots \ldots \ldots \ldots \ldots$ & 0.40 & 0.0006 & 350 & 0.71 \\
\hline 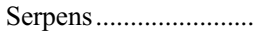 & 0.35 & 0.0139 & 800 & 2.36 \\
\hline
\end{tabular}

Note.-Best-fit values for the parameters used to convert IRIS flux to visual extinction.

a IRAS flux normalized to the 2MASS/NICER extinction.

b IRAS flux normalized to the Cambrésy extinction. 
possibility. We find that the best fit occurs between the emissionbased extinction map and the optical star count-based extinction map when the latter map has a constant value of 2.4 mag of visual extinction added to each pixel.

\section{BASIC FORMULAE}

The basic method that we use to calculate the dust color temperature and column density from the IRAS 60 and $100 \mu \mathrm{m}$ flux densities is similar to Wood et al. (1994) and Arce \& Goodman (1999b). The temperature is determined by the ratio of the 60 and $100 \mu \mathrm{m}$ flux densities. The column density of dust can be derived from either measured flux and the derived color temperature of the dust. The calculation of temperature and column density depends on the values of three parameters: two constants that determine the emissivity spectral index and the conversion from $100 \mu \mathrm{m}$ optical depth to visual extinction. We are able to solve for these parameters explicitly because we have an independent estimate of visual extinction, as explained in $\S 4$.

The dust temperature $T_{d}$ in each pixel of a FIR image can be obtained by assuming that the dust in a single beam is isothermal and that the observed ratio of 60 to $100 \mu \mathrm{m}$ emission is due to blackbody radiation from dust grains at $T_{d}$, modified by a powerlaw emissivity spectral index. The flux density of emission at a wavelength $\lambda_{i}$ is given by

$$
F_{i}=\left[\frac{2 h c}{\lambda_{i}^{3}\left(e^{h c /\left(\lambda_{i} k T_{d}\right)}-1\right)}\right] N_{d} \alpha \lambda_{i}^{-\beta} \Omega_{i},
$$

where $N_{d}$ represents column density of dust grains, $\alpha$ is a constant that relates the flux to the optical depth of the dust, $\beta$ is the emissivity spectral index, and $\Omega_{i}$ is the solid angle subtended at $\lambda_{i}$ by the detector.

Following Dupac et al. (2003), we use the equation

$$
\beta=\frac{1}{\delta+\omega T_{d}}
$$

to describe the observed inverse relationship between temperature and the emissivity spectral index. The parameters ( $\delta$ and $\omega)$ are derived separately for each cloud and subregion considered in this paper.

With the assumptions that the dust emission is optically thin at 60 and $100 \mu \mathrm{m}$ and that $\Omega_{60} \simeq \Omega_{100}$ (true for IRIS images), we can write the ratio, $R$, of the flux densities at 60 and $100 \mu \mathrm{m}$ as

$$
R=0.6^{-(3+\beta)} \frac{e^{144 / T_{d}}-1}{e^{240 / T_{d}}-1} .
$$

Once the appropriate value of $\beta$ is known, one can use equation (3) to derive $T_{d}$. The value of $\beta$ depends on such dust grain properties as composition, size, and compactness. For reference, a pure blackbody would have $\beta=0$, the amorphous layer-lattice matter has $\beta \sim 1$, and the metals and crystalline dielectrics have $\beta \sim 2$.

Once the dust temperature has been determined, the $100 \mu \mathrm{m}$ dust optical depth can be calculated as

$$
\tau_{100}=\frac{F_{\lambda}(100 \mu \mathrm{m})}{B_{\lambda}\left(100 \mu \mathrm{m}, T_{d}\right)},
$$

where $B_{\lambda}$ is the Planck function and $F_{\lambda}(100 \mu \mathrm{m})$ is the $100 \mu \mathrm{m}$ flux.

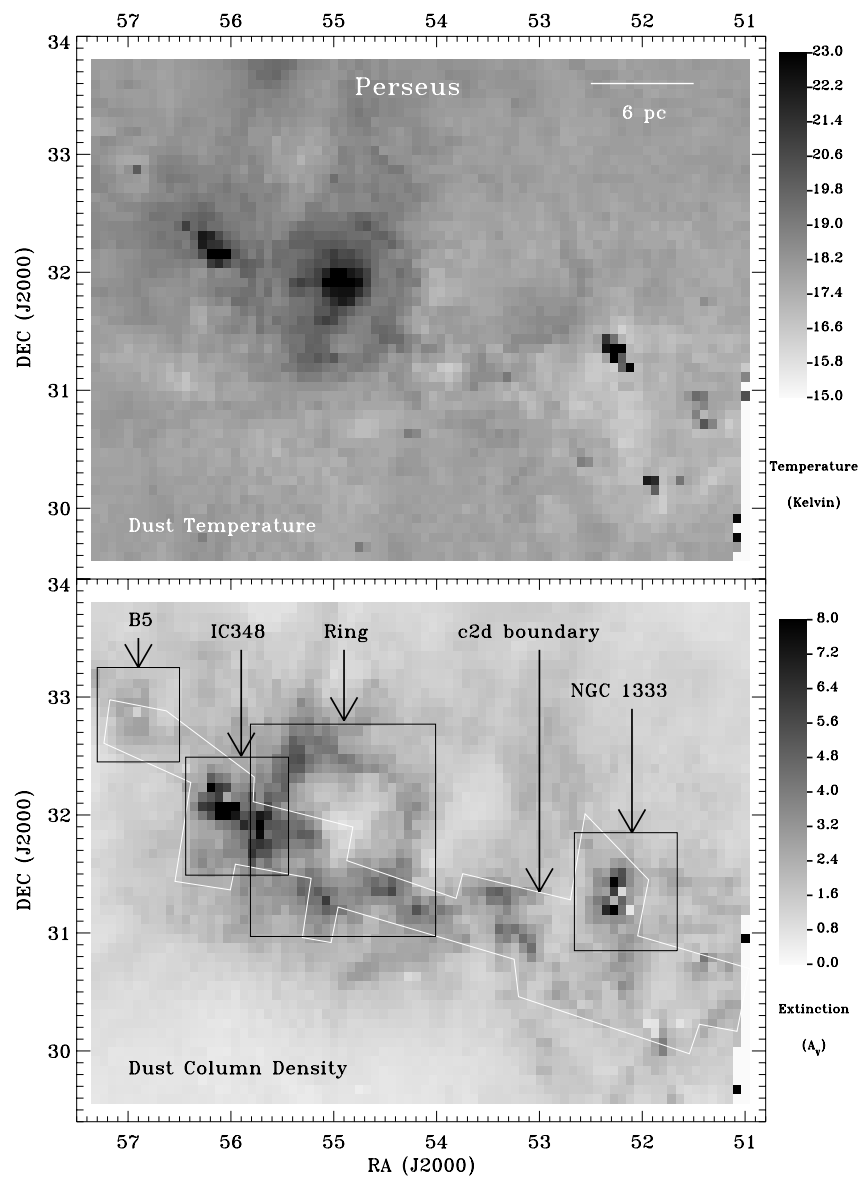

FIG. 2.-Dust color temperature map (top) and column density map (bottom) of Perseus, created from IRIS 60 and $100 \mu \mathrm{m}$ images. [See the electronic edition of the Journal for a color version of this figure.]

The $100 \mu \mathrm{m}$ optical depth can then be converted to $V$-band extinction using

$$
A_{V}=X \tau_{100}
$$

where $X$ is a parameter relating the thermal emission properties of dust to its optical absorption qualities.

\section{DERIVATION OF CONSTANTS}

We can derive the values of the three parameters $(\delta, \omega$, and $X)$ from the IRIS emission maps and extinction maps from Cambrésy (1999) and J. Alves et al. (2005, in preparation). Each optical and NIR extinction map described in $\S 2.2$ is used as a "model" extinction map to fit the IRIS-implied column density map using the three adjustable parameters that are explained in $\S 3$. The IDL task AMOEBA was used to simultaneously fit all three parameters with the downhill simplex method of Nelder \& Meade (1965). Each combination of these three parameters is used to determine the dust temperature and column density at each point in the map (using the formulae in $\S 3$ ). The parameter values determined by this method are those that create a FIR-based extinction map that best matches the NIR color excess or optical star count-derived extinction map. This is a statistical point-by-point match, not a spatial match to features in the NIR/optical extinction map. As explained in $\S 2.2$, we solve for a fourth parameter in Serpens, which is the zero level of the optical extinction map.

Each cloud is considered separately, so the values of $\delta, \omega$, and $X$ are different for each cloud. Because Ophiuchus has two 


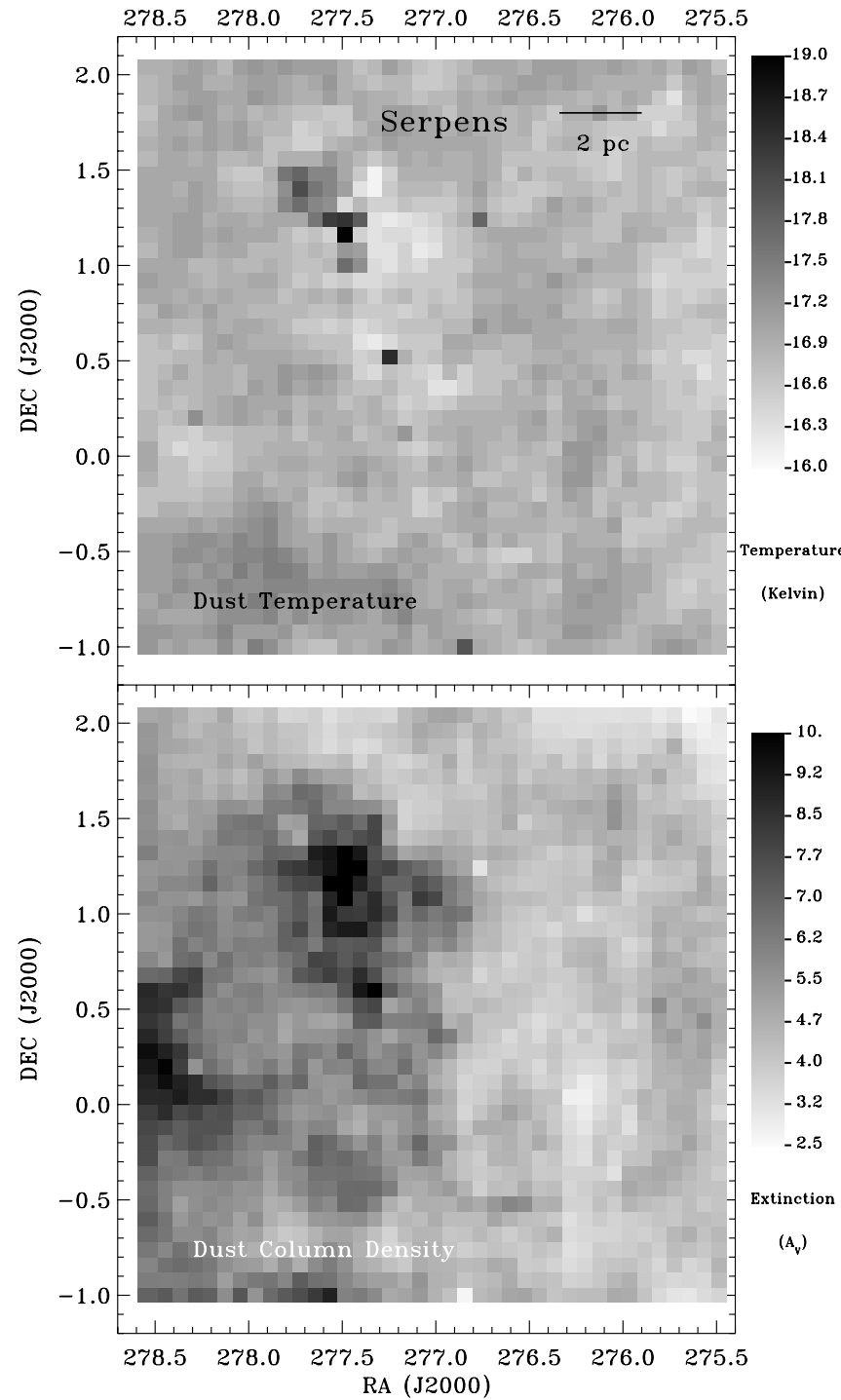

FIG. 3.-Dust color temperature map (top) and column density map (bottom) of Serpens, created from IRIS 60 and $100 \mu \mathrm{m}$ images. [See the electronic edition of the Journal for a color version of this figure.]

independent extinction maps, one from optical star counting (adjusted by the $0.71 \mathrm{mag}$ offset) and one from the NIR color excess method, it has two sets of constants derived for it. In this paper we assume that the values of the three parameters are constant within each image, although of course this does not have to be the case. For instance, it may be the case that areas of especially high or low column density do not share the same visual extinction conversion factor $(X)$. Our values for the three parameters for each region fitted (four in Serpens) are presented in Table 2.

\section{RESULTS AND DISCUSSION}

\subsection{Assumption of Thermal Equilibrium}

The derivations of dust temperature and column density from IRIS data rely on the assumption that the dust along each line of sight is in thermal equilibrium. However, the dust models of Désert et al. (1990) show that the emission at 60 and $100 \mu \mathrm{m}$ have contributions by big dust grains (BGs) and very small dust grains (VSGs). The VSGs are not in thermal equilibrium and emit mostly in the $60 \mu \mathrm{m}$ band, while the BGs are the primary contributors at $100 \mu \mathrm{m}$ and longer. Because of the emission from

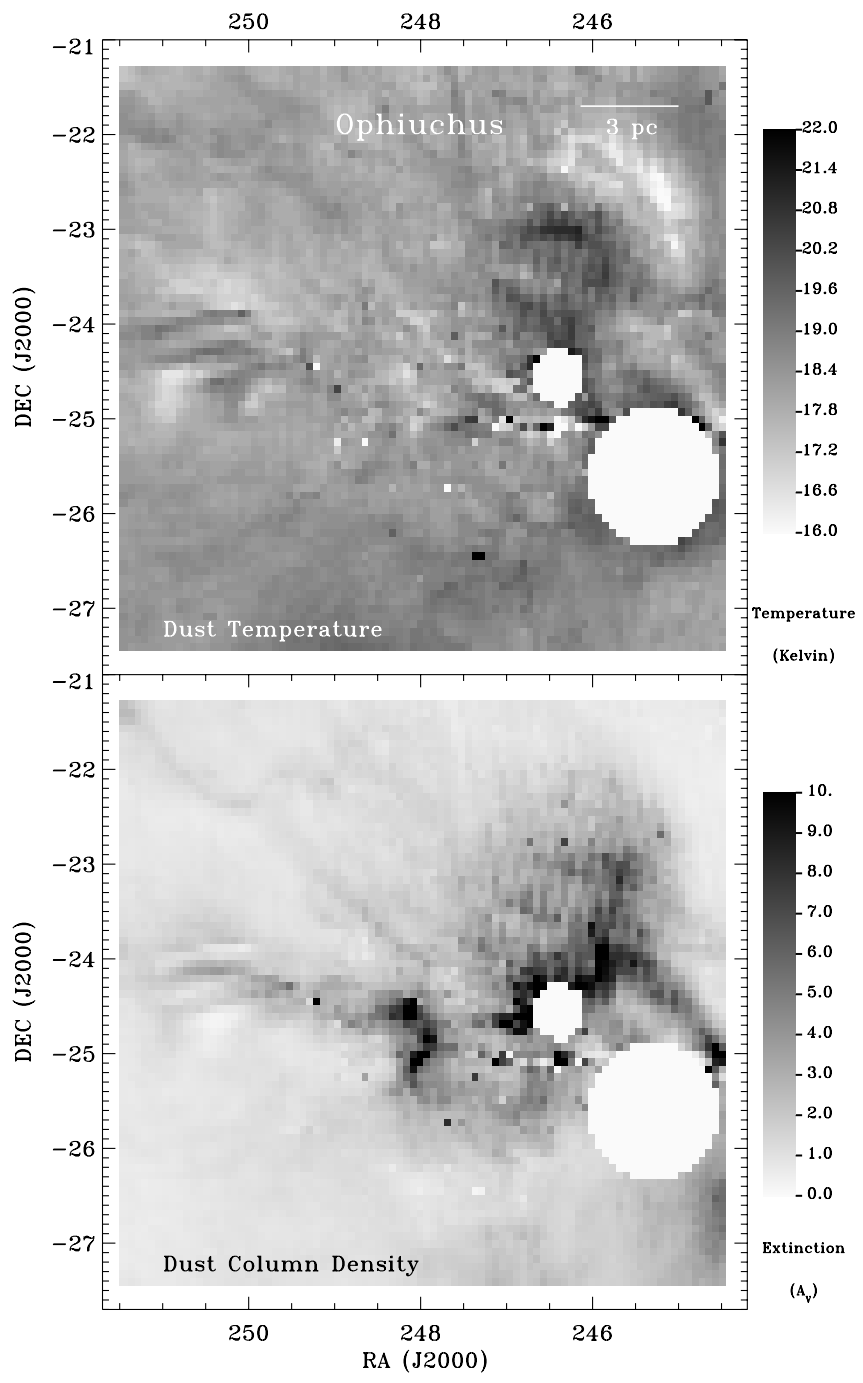

FIG. 4.-Dust color temperature map (top) and column density map (bottom) of Ophiuchus, created from IRIS 60 and $100 \mu$ m images. The two white circles have been excluded due to contamination from B stars. [See the electronic edition of the Journal for a color version of this figure.]

the VSGs, determining the temperature of the dust from the ratio of 60 and $100 \mu \mathrm{m}$ fluxes can yield temperatures that are systematically high. An empirical method for determining the color temperature and optical depth of dust using the 60 and $100 \mu \mathrm{m}$ bands from IRAS is presented in Nagata et al. (2002), but as this method has been calibrated for galaxies and not molecular clouds, we do not employ their method.

In order to remove the contribution of VSGs to the $60 \mu \mathrm{m}$ flux, we calibrate our temperature maps to those derived by SFD98, which are not corrupted by VSG emission because they used maps at $100 \mu \mathrm{m}$ and longer to derive their temperatures. We smooth the IRIS images to the resolution of the SFD98 temperature maps and calculate the temperature based on $100 \%$ of the $100 \mu \mathrm{m}$ flux and a lesser percentage of the $60 \mu \mathrm{m}$ flux, assuming that $\beta=2$ (as assumed by SFD 98). The fraction of the $60 \mu \mathrm{m}$ flux was chosen so as to best match our derived temperatures with the SFD98 temperatures. The remaining $60 \mu \mathrm{m}$ flux comes from the VSGs. Note that the temperatures we use in our calculations of column density do not assume that $\beta=2$; we use the temperature-dependent form of $\beta$ described in $\S 3$. However, the $60 \mu \mathrm{m}$ flux used in $\S 3$ is adjusted by this determination. By comparison to the SFD98 temperature maps, we find that the average VSG contribution to the 

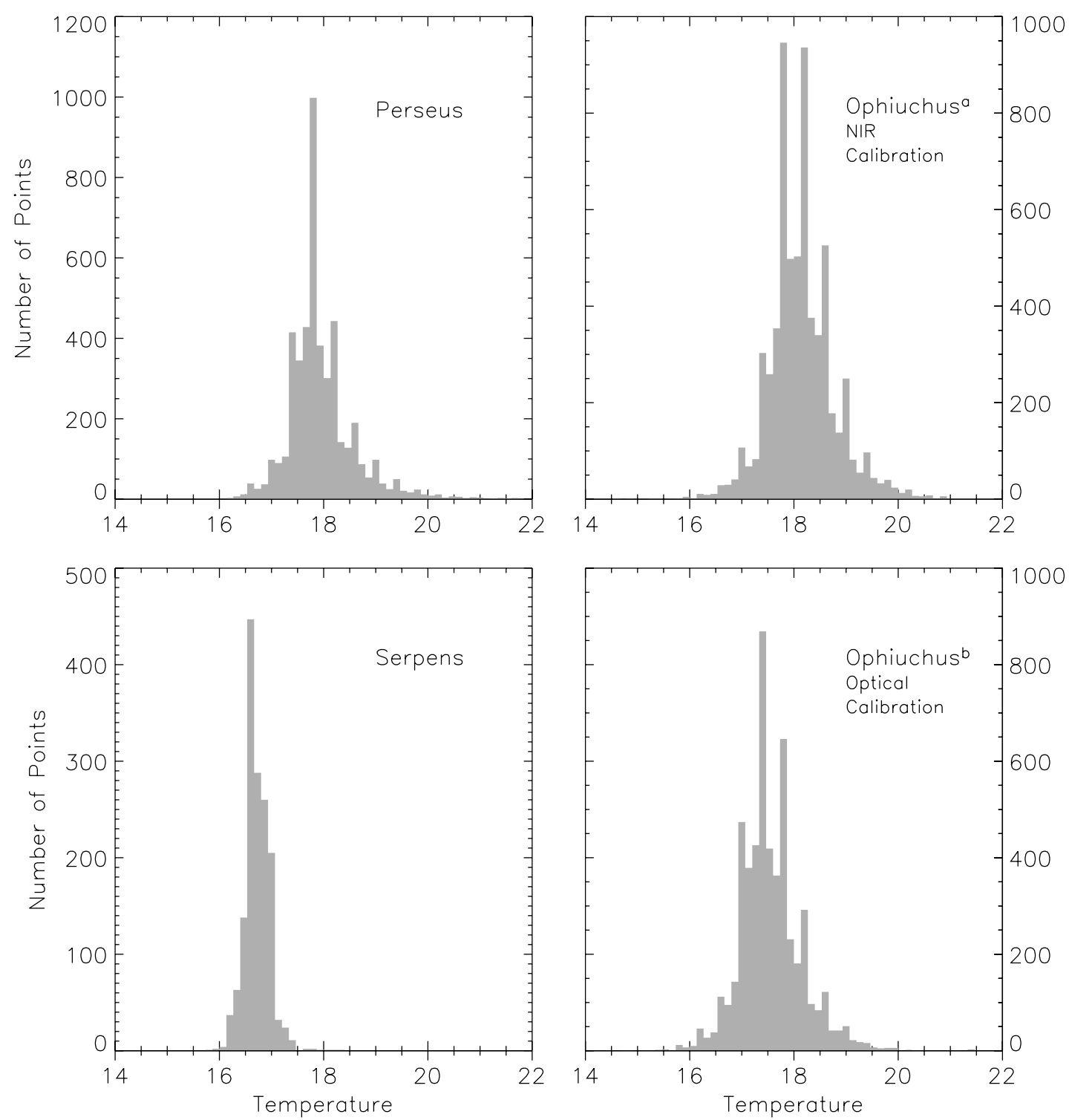

FIG. 5.-Filled histograms showing the temperature derived from the $60 / 100 \mu \mathrm{m}$ flux density ratio in Perseus, Serpens, and Ophiuchus after the calibration described in $\S 2$. The Ophiuchus ${ }^{\mathrm{a}}$ histogram (top right) is calibrated to the 2MASS extinction map. The Ophiuchus ${ }^{\mathrm{b}}$ histogram (bottom right) is calibrated to the Cambrésy data.

$60 \mu \mathrm{m}$ flux is $74 \%, 72 \%$, and $85 \%$ in Perseus, Ophiuchus, and Serpens, respectively.

\subsection{Assumption of Uniform Dust Properties}

The equations in this paper rely on the assumption that the dust along each line of sight is characterized by a single temperature, emissivity spectral index, and emissivity. This is a simplification that our method requires, although we recognize that real molecular clouds are much more complicated.

In their FIR study of interstellar cold dust in the Galaxy, Lagache et al. (1998) have found that there are at least two temperature components to the dust population. They find that the warmer component, associated with diffuse dust, has a temperature around $17.5 \mathrm{~K}$, while the colder component, associated with dense regions in the interstellar medium, has a temperature around $15 \mathrm{~K}$. The molecular clouds we study here are expected to have a range of temperatures along some lines of sight that is even wider than those seen in Lagache et al. (1998) because the Far Infrared Absolute Spectrophotometer (FIRAS) data used in this study has a beam size of $7^{\circ}$, which is larger than the size of the maps for each cloud studied in this paper. The color temperature that we derive from our isothermal assumption is therefore biased, as is the optical depth. This problem is especially relevant in Serpens, which is only a few degrees above the Galactic center, so there are certainly multiple environments integrated into each IRAS beam. A method for determining the amount of $100 \mu \mathrm{m}$ flux associated with the cold component of the BGs in a molecular cloud is explained in Abergel et al. (1994), Boulanger et al. (1998), and Laureijs et al. (1991). Nevertheless, Jarrett et al. (1989) find in Ophiuchus that there is a very tight linear correlation between FIR optical depth (determined in much the same way as presented here) and visual extinction for $A_{V} \leq 5$, so we trust that the errors introduced by our method are not prohibitively large.

\subsection{Temperature and Column Density Maps}

The dust color temperature and column density maps derived from our parameter fits and IRIS data are among the first publicly available data products distributed by the COMPLETE team. Temperature and extinction maps of Perseus, Ophiuchus, and 

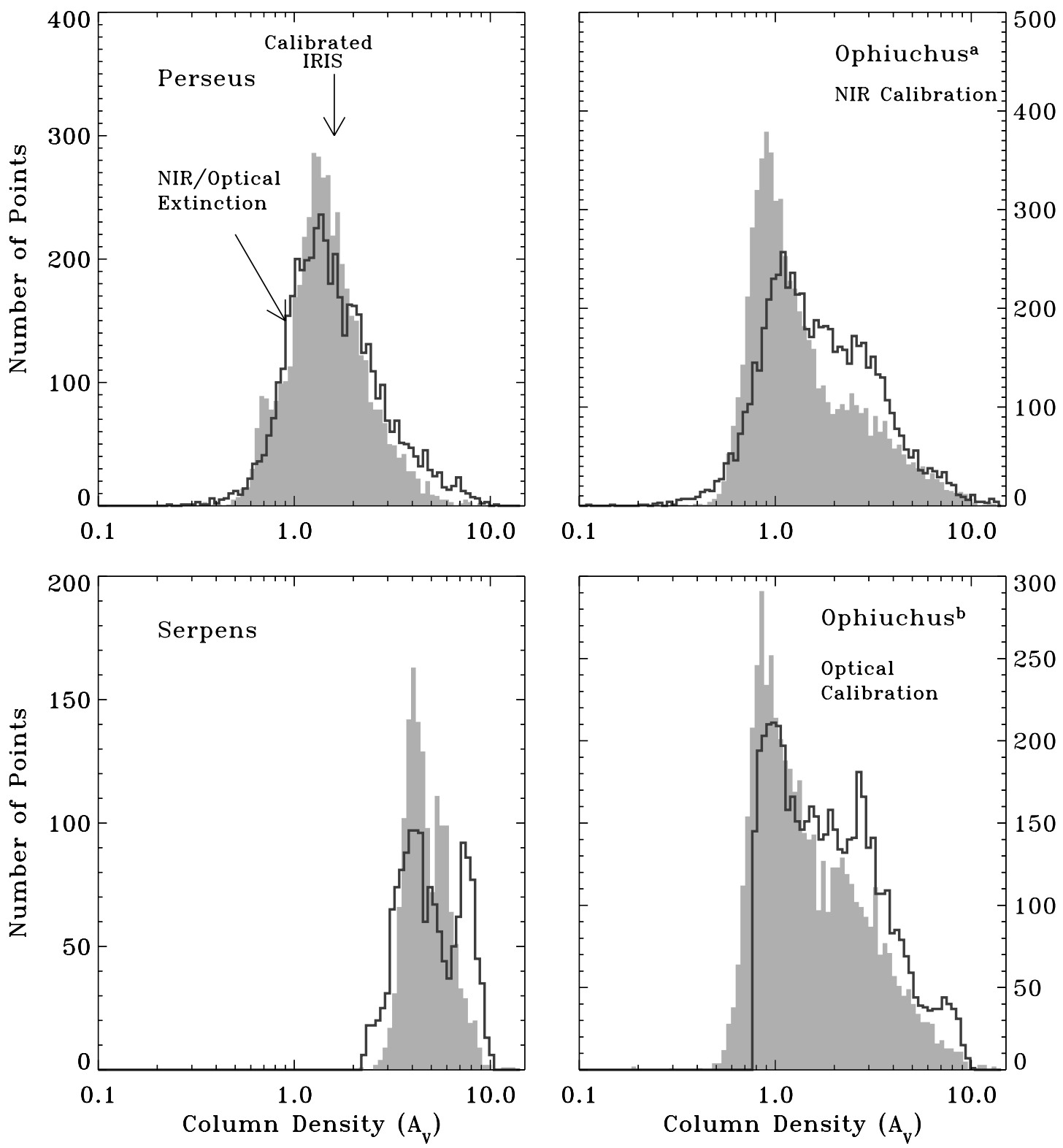

FIG. 6.-Filled histograms showing the extinction derived from the $60 / 100 \mu \mathrm{m}$ flux density ratio in Perseus, Serpens, and Ophiuchus after the calibration described in $\S 2$. The open histograms show the NIR/optical-derived extinction (from J. Alves et al. [2005, in preparation] and Cambrésy [1999]). The Ophiuchus ${ }^{\mathrm{a}}$ histogram (top right) is

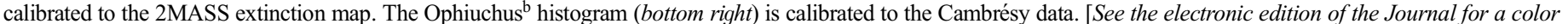
version of this figure.]

Serpens derived from the IRIS data are shown in Figures 2, 3, and 4. FITS files of these maps can be downloaded from the COMPLETE Web site. ${ }^{2}$

In Perseus, there is a striking ring of emission that is centered on a region of warm material. This ring has been discussed by Andersson et al. (2000), Pauls \& Schwartz (1989), and Fiedler et al. (1994) and is discussed in more detail in a companion paper to this work (Ridge et al. 2005). The Perseus ring does not stand out in the NICER extinction map as visibly as in the IRIS column density map because it is not a true column density feature. It is difficult to determine from emission maps alone whether individual features are the result of changing dust properties or column density enhancements.

A warm dust ring is evident in Ophiuchus in the temperature map, centered at the position R.A. $=16^{\mathrm{h}} 25^{\mathrm{m}} 35^{\mathrm{s}}$, decl. $=$

\footnotetext{
${ }^{2}$ See http://cfa-www.harvard.edu/COMPLETE.
}

$-23^{\circ} 26^{\prime} 50^{\prime \prime}$ (J2000.0). This ring was reported by Bernard et al. (1993) in a discussion of the FIR emission from Ophiuchus and Chameleon, but they did not investigate its nature or possible progenitor. The B star $\rho$ Oph and a number of X-ray sources are projected to lie within this shell of heated gas. The Ophiuchus ring will be further discussed in a future paper (D. Li et al. 2005, in preparation).

\subsection{Temperature and Extinction Distributions}

In Figure 5 we show histograms of the dust temperature in Perseus, Serpens, and Ophiuchus. Each distribution peaks near $17 \mathrm{~K}$, and all except Serpens have a spread of several degrees. The dust temperatures that we derive are, to an extent, calibrated with those derived by SFD98 (see our $\S 5.1$ ).

For Taurus, Arce \& Goodman (1999b) have shown that using IRAS 60 and $100 \mu \mathrm{m}$ flux densities to determine dust column density gives results consistent with other methods, such as the color 


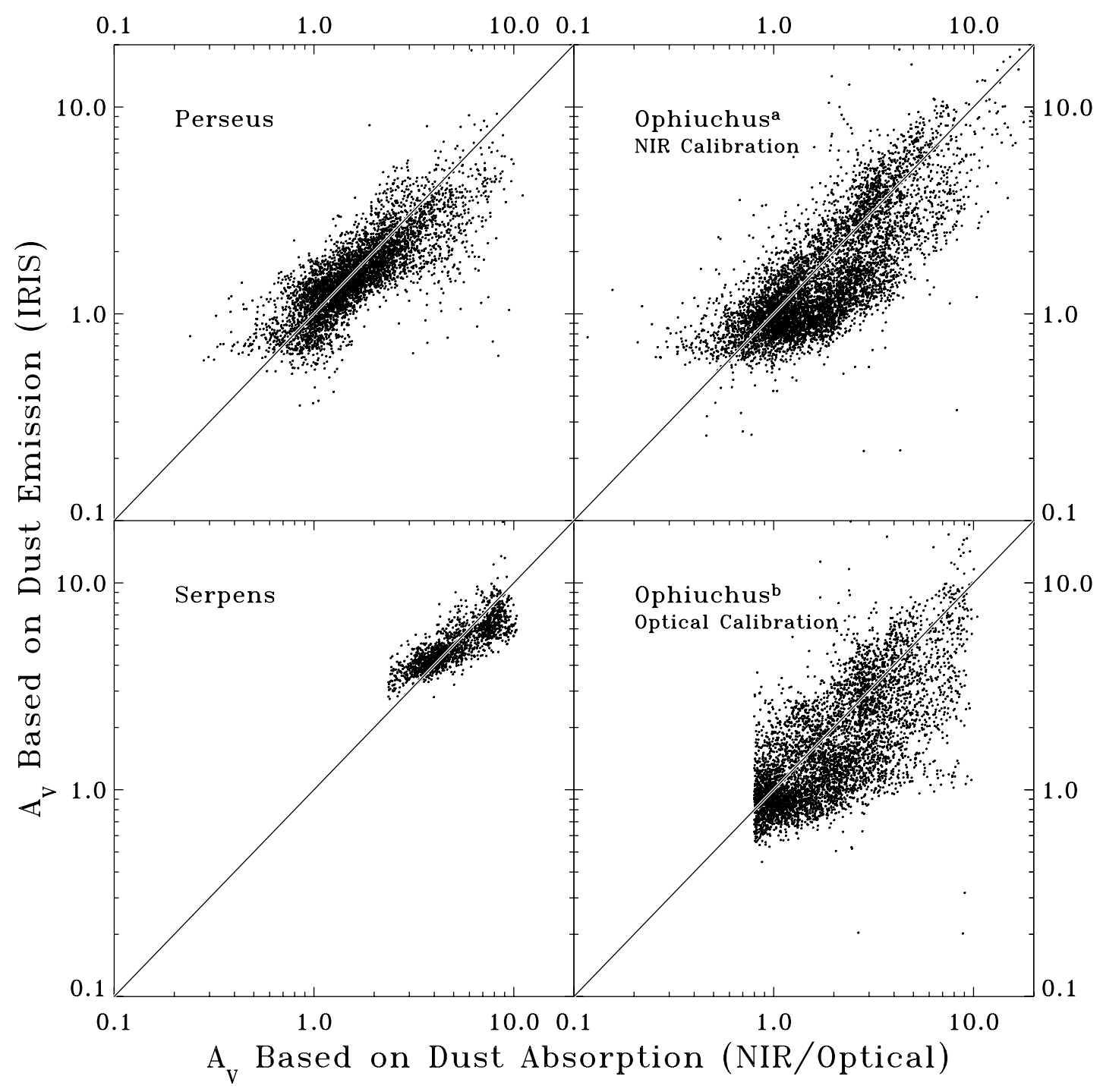

Fig. 7. - Scatter plot of the corrected IRIS extinction (derived from the $60 / 100 \mu \mathrm{m}$ flux density ratio in Perseus, Serpens, and Ophiuchus) and the model extinction (from J. Alves et al. [2005, in preparation] and Cambrésy [1999]). The Ophiuchus ${ }^{\mathrm{a}}$ IRIS extinction (top right) is calibrated to the 2MASS extinction map. The Ophiuchus ${ }^{\mathrm{b}}$ IRIS extinction (bottom right) is recalibrated to the Cambrésy data. Note that the data are shown here in log-log plots for clarity of display, but all the fitting is of linear (not log) quantities. The sharp cutoff in the Ophiuchus ${ }^{\mathrm{b}}$ panel is a result of having added 0.71 mag to the optically derived extinction. The sharp cutoff in the Serpens panel is a result of having added $2.2 \mathrm{mag}$ to the optically derived extinction. [See the electronic edition of the Journal for a color version of this figure.]

excess method (e.g., NICE/NICER), star counts (e.g., Cambrésy 1999), and using an optical ( $V$ and $R$ ) version of the average color excess method used by Lada et al. (1994). Here we compare the IRIS-derived extinction maps of Perseus, Serpens, and Ophiuchus with maps created by some of these other methods.

Our method requires the FIR-derived extinction to best match the NIR- or optical-derived extinction, and the global agreement can be seen in Figure 6. However, the point-to-point extinction values can be significantly different between the two methods, as shown in Figure 7. The large scatter in these extinction plots ( $1 \sigma \simeq 1 \mathrm{mag} A_{V}$ ) is likely the result of the various assumptions used in our calculations. For instance, it is unlikely to be the case that all of the dust along a given line of sight can be well characterized by a single temperature or emissivity spectral index, especially along lines of sight through the denser regions of the molecular clouds. It is also likely that the dust optical depth to visual extinction conversion factor $(X)$ is not constant throughout a cloud volume. The flux detected by IRAS comes preferentially from warmer dust, while the extinction maps made from NIR and optical data have no temperature bias, so it is also pos- sible (and in many regions likely) that the dust doing most of the FIR emitting is not the same dust responsible for most of the extinguishing at shorter wavelengths. The scatter for each cloud is shown in Table 3.

As a test to see whether the parameters determined for one cloud can be successfully used to describe another, we used the Perseus fits for $\delta, \omega$, and $X$ for the Ophiuchus 60 and $100 \mu \mathrm{m}$ IRIS maps and compared the derived extinction to the 2MASS extinction map of Ophiuchus. The median point-to-point difference was 0.8 mag of visual extinction, with a standard deviation of 1.9 mag. When the optimized parameters for Ophiuchus are used, the median point-to-point difference is only $0.2 \mathrm{mag}$, with a scatter of 1.2 mag. We conclude that the fits for one cloud are unlikely to be appropriate for other clouds, and therefore caution should be used in attempts to estimate extinction within molecular clouds from IRAS emission maps alone.

We have derived separate values for $\delta, \omega$, and $X$ for various regions within Perseus to see if the dust properties are significantly different there than in the cloud as a whole. The regions considered were B5, IC 348, NGC 1333, the emission ring, and 
TABLE 3

Scatter between IRAS Implied Extinction and NIR/Optical-derived Extinction

\begin{tabular}{|c|c|c|c|c|}
\hline Cloud & $\begin{array}{l}\text { Median Difference } \\
\quad\left(\operatorname{mag} A_{V}\right)\end{array}$ & $\begin{array}{c}1 \sigma \text { Scatter } \\
\text { between Methods } \\
\left(\operatorname{mag} A_{V}\right)\end{array}$ & $\begin{array}{l}\text { Scatter between } \\
\text { Methods } \\
(\%)\end{array}$ & $\begin{array}{c}\tau_{100} / A_{V} \\
\left(10^{-4} \mathrm{mag}^{-1}\right)\end{array}$ \\
\hline 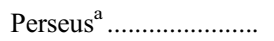 & -0.1 & 0.9 & 30 & $9 \pm 3$ \\
\hline Ophiuchus $^{\mathrm{a}} \ldots \ldots \ldots \ldots \ldots \ldots$ & -0.2 & 1.2 & 50 & $17 \pm 9$ \\
\hline 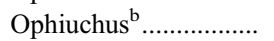 & -0.2 & 1.4 & 50 & $23 \pm 13$ \\
\hline 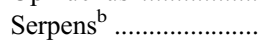 & 0.0 & 1.2 & 20 & $12 \pm 3$ \\
\hline
\end{tabular}

NoTE.-Median difference between the different ways to estimate extinction within a molecular cloud (IRIS $A_{V}$-NIR/optical $A_{V}$ ) and the scatter between the methods (see $\S 5.4$ ).

a IRAS flux normalized to the 2MASS/NICER extinction.

b IRAS flux normalized to the Cambrésy extinction.

the area surveyed by the c2d Spitzer Legacy project. The locations and sizes of the Perseus subregions are shown in Table 1. The values of $\delta, \omega$, and $X$ for these subregions are shown in Table 2 and displayed in Figure 2. The value of $\delta$ varies by about $10 \%, \omega$ varies by about $20 \%$, and $X$ varies by $20 \%$ in these subregions of Perseus. NGC 1333 varies much more significantly from the other subregions of Perseus.

We conclude that the method described here for converting dust emission to visual extinction can be used with confidence to find regions with high or low extinction and to determine the average extinction in large areas $\left(\sim 0.25 \mathrm{deg}^{2}\right)$. However, the extinction determined in any individual $5^{\prime}$ pixel should not be trusted to represent the true absolute extinction to better than $\sim 2.5 \mathrm{mag}(2 \sigma)$ of visual extinction. This is made clear by the significant variability in the emissivity spectral index of dust and the conversion from dust optical depth to visual extinction between clouds and within clouds, as shown in Tables 2 and 3.

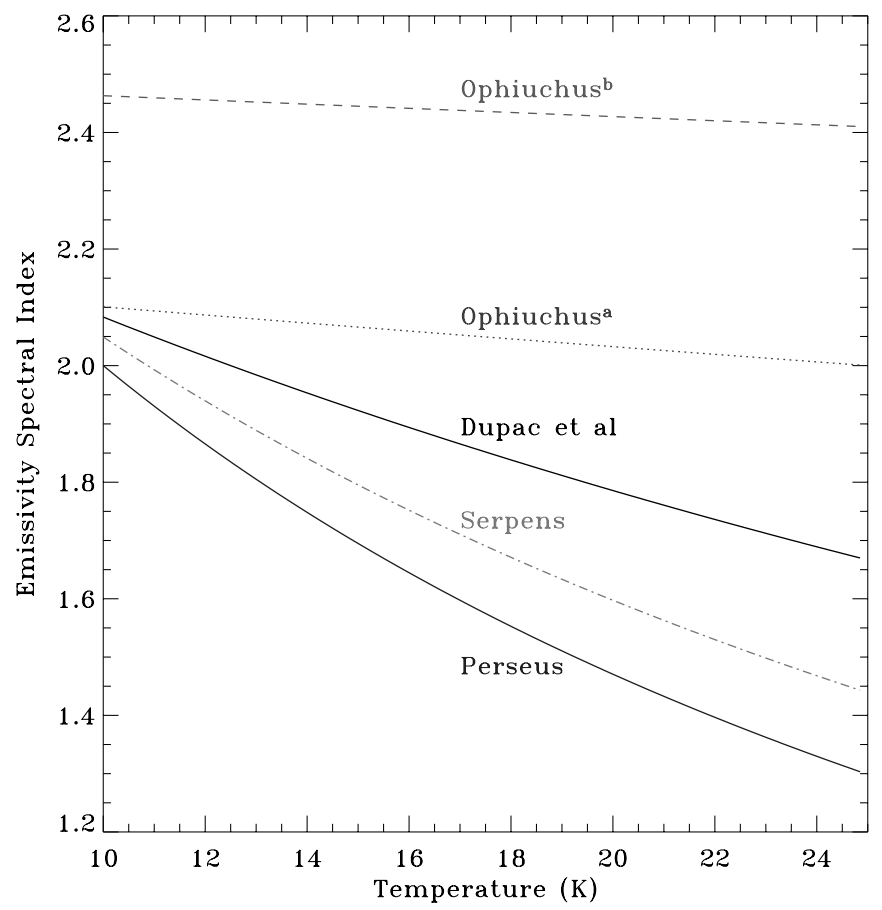

FIG. 8.-Plot of the emissivity spectral index vs. temperature for Perseus, Ophiuchus, and Serpens. The Ophiuchus ${ }^{\mathrm{a}}$ curve is for the IRIS data calibrated to the 2MASS extinction map. The Ophiuchus ${ }^{\mathrm{b}}$ curve is calibrated to the Cambrésy data. The "Dupac et al." curve is the best-fit curve to a collection of FIR/submillimeter emission regions as presented in Dupac et al. (2003). [See the electronic edition of the Journal for a color version of this figure.]

\subsection{Emissivity Spectral Index}

The emissivity spectral index that we use in this paper varies with temperature as shown in equation (2). The values that we find for the parameters $\delta$ and $\omega$ are shown in Table 2. Their values as determined by Dupac et al. (2003) are 0.4 and 0.008, respectively, which comes from a much broader range of environments and many more measurements of the FIR/submillimeter flux. Figure 8 shows the emissivity spectral index that we find for each cloud plotted along with the curve from Dupac et al. (2003). The emissivity spectral index is considerably larger in Ophiuchus than in Serpens, which is somewhat higher than $\beta$ in Perseus. The best-fit curve to the wider range of environments in Dupac et al. (2003) falls between our Serpens and Ophiuchus curves.

\section{SUMMARY}

We have described a new method that uses NIR color excesses or optical star counts to constrain the conversion of IRIS 60 and $100 \mu \mathrm{m}$ data into color temperature and column density maps. Our method also derives the dust emissivity spectral index and the conversion from dust $100 \mu \mathrm{m}$ optical depth to visual extinction. We test the IRIS 60 and $100 \mu \mathrm{m}$ zero points and confirm that, unlike earlier releases of IRAS data, the IRIS recalibration is properly zero-point-corrected. We find that the very small grain contribution to the $60 \mu \mathrm{m}$ flux is significant.

The dust temperature maps of Perseus, Serpens, and Ophiuchus available through the COMPLETE Web site should be the best dust temperature maps of large star-forming regions at $5^{\prime}$ resolution created to date. The dust temperatures derived here are dependent on the emissivity spectral index of the dust, which is solved globally for each molecular cloud, and recorded in Table 2.

Our work here indicates that one cannot confidently convert dust $100 \mu$ m optical depth to visual extinction without the benefit of having an extinction map made in an alternative manner to use as a model because the conversion constant $X$ varies significantly from cloud to cloud, and even within a cloud (see Table 2). Using the values for the dust emissivity spectral index and visual extinction conversion derived for one cloud results in a significant miscalculation of the extinction in other clouds. Even with the NICER and optical star counting extinction maps used here to calibrate the IRAS data, there is significant point-to-point variation between the two estimates (see Fig. 1). In fact, we have shown that in Ophiuchus the optical star count method employed by Cambrésy (1999) has an offset from the NICER method applied to the 2MASS data set of $\sim 0.71$ mag in $A_{V}$, with a scatter of $0.7 \mathrm{mag}$, so the issue is not simply one of dealing with the FIR dust properties in molecular clouds. In addition, we have identified two ring structures - one in Perseus and one in 
Ophiuchus - that are discussed in upcoming papers (Ridge et al. [2005] and D. Li et al. [2005, in preparation], respectively).

We would like to thank the referee Laurent Cambrésy for many useful comments. This research has made use of the NASA IPAC Infrared Science Archive, which is operated by the Jet Propulsion Laboratory, California Institute of Technology, under contract with the National Aeronautics and Space Administration. This publication makes use of data products from the Two Micron All Sky Survey (2MASS), which is a joint project of the University of Massachusetts and the Infrared Processing and Analysis Center/ California Institute of Technology, funded by the National Aeronautics and Space Administration and the National Science Foundation. This material is based on work supported under a National Science Foundation Graduate Research Fellowship.
Abergel, A., Boulanger, F., Mizuno, A., \& Fukui, Y. 1994, ApJ, 423, L59 Andersson, B.-G., Wannier, P. G., Moriarty-Schieven, G. H., \& Bakker, E. J. 2000, AJ, 119, 1325

Arce, H. G., \& Goodman, A. A. 1999a, ApJ, 512, L135 . 1999b, ApJ, 517, 264

Bernard, J. P., Boulanger, F., \& Puget, J. L. 1993, A\&A, 277, 609

Boulanger, F., Bronfman, L., Dame, T. M., \& Thaddeus, P. 1998, A\&A, 332, 273

Cambrésy, L. 1999, A\&A, 345, 965

Cambrésy, L., Jarrett, T. H., \& Beichman, C. A. 2005, A\&A, 435, 131

Désert, F.-X., Boulanger, F., \& Puget, J. L. 1990, A\&A, 237, 215

Dupac, X., et al. 2003, A\&A, 404, L11

Evans, N. J., et al. 2003, PASP, 115, 965

Fiedler, R., Pauls, T., Johnston, K. J., \& Dennison, B. 1994, ApJ, 430, 595

Goodman, A. A. 2004, in ASP Conf. Ser. 323, Star Formation in the Interstellar Medium: In Honor of David Hollenbach, Chris McKee, and Frank Shu, ed. D. Johnstone et al. (San Francisco: ASP), 171

\section{REFERENCES}

Jarrett, T. H., Dickman, R. L., \& Herbst, W. 1989, ApJ, 345, 881

Lada, C. J., Lada, E. A., Clemens, D. P., \& Bally, J. 1994, ApJ, 429, 694

Lagache, G., Abergel, A., Boulanger, F., \& Puget, J.-L. 1998, A\&A, 333, 709

Laureijs, R. J., Clark, F. O., \& Prusti, T. 1991, ApJ, 372, 185

Lombardi, M., \& Alves, J. 2001, A\&A, 377, 1023

Miville-Deschênes, M., \& Lagache, G. 2005, ApJS, 157, 302

Monet, D. 1996, BAAS, 188, 54.04

Nagata, H., Shibai, H., Takeuchi, T. T., \& Onaka, T. 2002, PASJ, 54, 695

Nelder, J., \& Meade, R. 1965, Comput. J., 7, 308

Pauls, T., \& Schwartz, P. R. 1989, in The Physics and Chemistry of Interstellar Molecular Clouds, ed. G. Winnewisser \& J. T. Armstrong (Berlin: Springer), 225

Ridge, N., Schnee, S., \& Goodman, A. A. 2005, ApJ, submitted Schlegel, D. J., Finkbeiner, D. P., \& Davis, M. 1998, ApJ, 500, 525 Wood, D. O. S., Myers, P. C., \& Daugherty, D. A. 1994, ApJS, 95, 457 